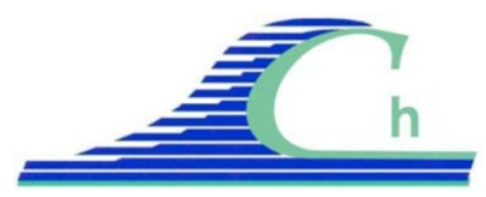

XII ${ }^{\text {ìmes }}$ Journées Nationales Génie Côtier - Génie Civil

Cherbourg, 12-14 juin 2012

DOI:10.5150/jngcgc.2012.106-A @ Editions Paralia CFL

disponible en ligne - http://www.paralia.fr - available online

\title{
Les variantes granulats dans le contexte des îles océaniennes affectées par le changement climatique. Application au cas de l’île de Wallis (Pacifique sud-ouest)
}

\author{
Michel ALLENBACH ${ }^{1}$, Thierry HOIBIAN $^{1}$
}

1. Université de la Nouvelle-Calédonie, PPME - EA 3325, BP R4, 98800 Nouméa, Nouvelle Calédonie.

allenbach@univ-nc.nc;hoibian@univ-nc.nc)

\section{Résumé :}

Les incidences majeures des changements climatiques futurs sur les petites îles océaniennes annoncées par le GIEC (2007) sont l'intensification des inondations, des tempêtes et de l'érosion, l'atteinte aux ressources locales, le blanchissement des coraux et la réduction des ressources en eau. Ces tendances sont amplifiées par les erreurs anthropiques : poldérisation du littoral, prélèvements anarchiques de sables et graviers sur les plages et dunes. La communication se propose de faire le point, à partir d'une monographie régionale consacrée au cas de l'archipel des îles Wallis (Pacifique sudouest) sur les variantes utilisables pour pallier le manque récurrent de granulats sur les espaces insulaires océaniens. Ces variantes, par ailleurs classiques, doivent être étudiées en regard, d'une part, de leur impact environnemental sur un écosystème fragile, le milieu récifal et d'autre part, des réalités socio-économiques et techniques de ces espaces isolés au sein de l'océan Pacifique.

\section{Mots-clés :}

Granulats - Changement climatique global - Wallis et Futuna - Pacifique sud-ouest Ecosystème récifal - Développement durable - Scories de nickel

\section{Abstract:}

The major impacts of future climate change on small Pacific islands announced by the IPCC (2007) are the increase in floods, storms and erosion, damage to local resources, coral bleaching and reduced resources water. These tendencies are amplified by human error : polders, unregulated gathering sand and gravel on beaches and dunes. The paper aims to take stock, from a regional monograph devoted to the case of the Wallis archipelago (Southwest Pacific) the variants can be used to offset the recurrent lack of aggregates on the Pacific island areas. These variants, also classic, are to be studied with their environmental impact on a fragile ecosystem : the reef on one hand and socioeconomic and technical issues of the isolated areas within the Pacific Ocean on the other.

\section{Keys words:}

Aggregates - Global Climate Change - Wallis and Futuna - Pacific Southwest - Reef ecosystems - Sustainable Development - Nickel Slag 


\section{Présentation générale de la problématique}

\subsection{Cadre géographique}

La problématique traitée concerne la zone Pacifique sud-ouest (figure 1) qui compte trois territoires ultramarins français dont celui de Wallis et Futuna qui est l'objet principal de la communication présentée. Les îles Wallis sont situées par $13^{\circ} 16^{\prime}$ de latitude sud et $176^{\circ} 15^{\prime}$ de longitude est. Essentiellement basaltique, l'île principale, Uvéa, de $15 \mathrm{~km}$ sur 6, est entourée d'un lagon de 3 à $5 \mathrm{~km}$ de largeur. Ce dernier est ceinturé par un récif barière quasi continu d'une soixantaine de $\mathrm{km}$ de longueur percé d'une passe principale au sud. Le récif barrière constitue, pour l'espace lagonaire, un rempart efficace contre les houles océaniques qui brisent à son contact. L’hydrodynamisme très modéré du milieu lagonaire, hormis en période cyclonique (le dernier ayant touché l'île est Thomas, en 2010) n’est pas susceptible de générer les phénomènes érosifs notés sur l'ensemble du linéaire littoral de l'île et les causes sont à rechercher pour l'essentiel dans des actions anthropiques qui seront commentées dans les alinéas suivants.

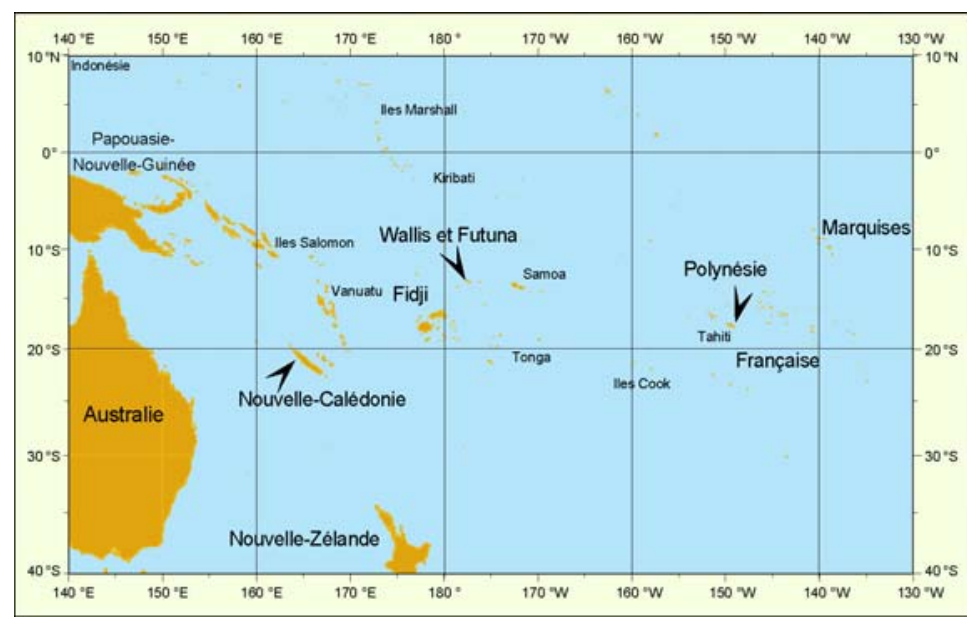

Figure 1. Le sud-ouest Pacifique. Présentation régionale

1.2 L’impact cumulé du changement global et de l'anthropisation des sites sur l'évolution du trait de côte

Les incidences des changements climatiques futurs sur les petites îles océaniennes, (GIEC, 2007), sont l'intensification des inondations, des ondes de tempêtes et de l'érosion menaçant les infrastructures, les établissements humains et les installations vitales pour les populations insulaires. Il s'y ajoute l'atteinte aux ressources locales du fait de l'érosion des plages et du blanchissement des coraux et la réduction des ressources en eau. Ces tendances sont amplifiées par les erreurs anthropiques : ouvrages de génie civil défaillants, poldérisation du littoral, prélèvements de granulats de plages 


\section{XII ${ }^{\text {èmes }}$ Journées Nationales Génie Côtier - Génie Civil \\ Cherbourg, 12-14 juin 2012}

et de dunes littorales (ALLENBACH \& HOIBIAN, 2008), erreurs génériques à l'ensemble de l'espace insulaire océanien.

\subsection{Les ressources en granulats}

La recherche de granulats pour les nouvelles constructions (passage de l'habitat traditionnel aux maisons en dur), les zones à remblayer sur l'espace maritime, les corps de chaussée et les défenses contre l'érosion marine sont les principaux besoins exprimés. L'extraction "sauvage" des granulats de plage permet aux populations de satisfaire leurs besoins personnels et leur assure des rentrées monétaires importantes par la vente du sable aux entrepreneurs locaux. La pratique se traduit par la disparition progressive des plages et la destruction des dunes sur les rares segments littoraux où ces formations sédimentaires sont encore présentes. Sur les 30 dernières années, des prélèvements sauvages de plusieurs dizaines de milliers de $\mathrm{m}^{3}$ ont ainsi été réalisés sur les zones sud-ouest (secteur d'Utuleve), sud (secteur de Halalo) et nord-ouest (secteur de Fakapepe) du littoral. Sur ce dernier secteur choisi à titre d'exemple, l'analyse diachronique de l'imagerie aérienne disponible (1982-2008) présentée à la figure 2 a permis de quantifier à environ $27000 \mathrm{~m}^{2}$ l'emprise surfacique sur laquelle le sable a été enlevé.
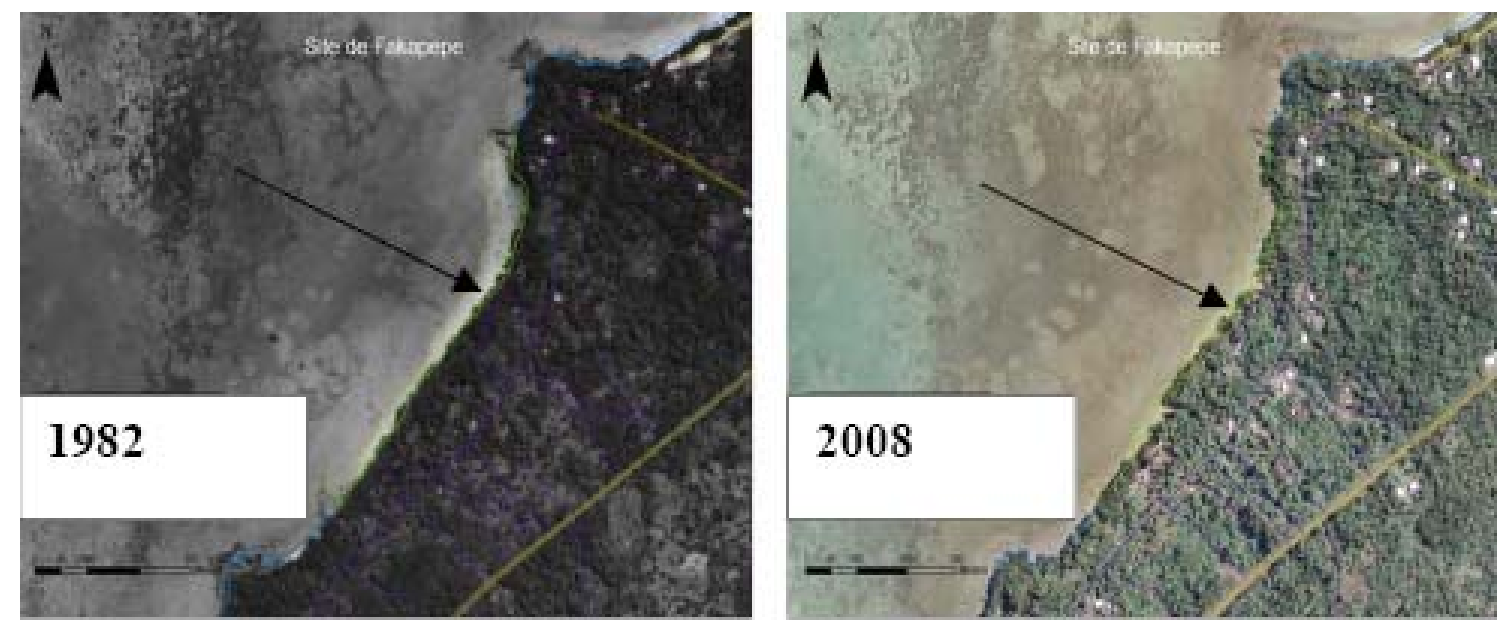

Figure 2. Evolution diachronique du secteur littoral de Fakapepe (Secteur littoral nord-ouest de l'île de Wallis)

Il s'agit là de dégats environnementaux sérieux qui touchent l'ensemble des plages de Wallis et des îlots du lagon (BANTOS, 2011). La recherche de solutions doit tenir compte des contraintes de la vision océanienne de l'espace littoral (WORLICZEK 2011), du manque d'outils juridiques applicables pour lutter contre les activités anthropiques déstabilisatrices du littoral (notion de domaine public maritime absente, loi littoral non applicable, etc.) et des réalités économiques d'un territoire où les ressources monétaires sont faibles. Sur financement de l'IFRECOR (Initiative française pour les 
récifs coralliens), une expertise a été réalisée (ALLENBACH, 2012) pour faire le point sur les trois variantes potentiellement utilisables en matière de granulats sur Wallis : i) le concassage de matériaux terrestres, ii) l'utilisation des sables lagonaires, iii) l'importation de matériaux exogénes. Il s'agit de trouver le moyen de répondre aux besoins exprimés et d'essayer de trouver des produits de substitution aux sables de plage.

\section{Les variantes granulats envisageables}

\subsection{Le concassage des matériaux terrestres basaltiques}

Il existe une filière de production de granulats à partir de l'extraction des matériaux basaltiques en carrière qui permet de fournir toutes les granulomètries depuis des blocs de 2,5 t (limite haute des besoins exprimés sur l'île) jusqu'à 300 micromètres (granulométrie souhaitable pour faire des corps de chaussée). Parallèlement à la production des granulats, l'exploitant élabore un produit dérivé : des parpaings préfabriqués (1000 unités/jour). Les principaux clients du carrier sont l’Etat et le Territoire par l'intermédiaire du Service des Travaux Publics. Les produits sont essentiellement destinés à satisfaire la demande exprimée pour l'aménagement littoral (port, digue et cordons d'enrochement) en ce qui concerne les enrochements et pour la confection des chaussées pour les petites granulométries produites. Le volume produit par le carrier est estimé à $250 \mathrm{~m}^{3}$ de graves traitées/jour. La ressource est suffisante pour couvrir les besoins classiques exprimés et l'impact environnemental est faible. La variante est performante et elle occasionne peu de désordres environnementaux, mais en regard du problème de l'extraction sauvage des granulats de plage, les coûts de production au sortir de la centrale à granulats ne permettent pas à la plus grande partie de la population d'y avoir recours. La production de sable dérivé du concassage des basaltes est de l'ordre de $60 \mathrm{~m}^{3}$ par semaine, mais pourrait être bien supérieure si la pratique de l'extraction "sauvage" des sables de plage et de dunes n'était pas autorisée. Théoriquement interdite, elle est de fait quasi systématiquement utilisée, même parfois sur des marchés d'Etat où la vigilance des contrôles est souvent contournée par des entrepreneurs sans grands scrupules. La résolution du problème de l'extraction sauvage est fondamentalement un problème économique et l'analyse des diverses variantes techniques utilisables pour réduire les dysfonctionnements environnementaux associés au marché des granulats doit déboucher sur des propositions concrètes. La valeur économique des milieux naturels est aujourd'hui une notion qui émerge fortement dans le cadre des politiques de développement durable. Les îles de Wallis et Futuna, milieux insulaires fragiles, ne peuvent pas faire l'impasse d'une analyse socio-économique et politique du sujet, débouchant sur des solutions novatrices de compensation financière des milieux naturels "sauvegardés". Il ne sert à rien que des politiques publiques financent de la lutte contre l'érosion (murs et cordons d'enrochements) si ces mêmes 


\section{XII ${ }^{\text {èmes }}$ Journées Nationales Génie Côtier - Génie Civil \\ Cherbourg, 12-14 juin 2012}

politiques contribuent à amplifier l'érosion par ailleurs, en ne prenant pas les décisions qui s'imposent pour limiter, voire annuler les dysfonctionnements constatés.

\subsection{L'extraction des sables lagonaires}

Il s'agit d'une solution assez largement employée en Océanie. A titre d'exemple, pour illustrer l'actualité très forte du sujet pour la région, nous citerons le programme ESAT (Environmentally Sustainable Aggregates for Tarawa) que mène la SOPAC (SouthOuest Pacific Applied Geosciences Commission) sur le Territoire de la République de Kiribati. Il s'agit d'un programme qui bénéficie d'un financement européen (EDF9) de 2,2 millions d'euros. Il est mené de façon partenariale entre l'OIP (Ocean \& Islands Program) de la SOPAC et le gouvernement de Kiribati. Le programme ESAT étudie la faisabilité de mise en exploitation d'une ressource d'agrégats marins lagonaires (volume extrait envisagé $=70000 \mathrm{~m}^{3} /$ an) destinés à remplacer les prélèvements de sable qui sont extraits chaque année de la partie sud de l'atoll de Tarawa. En matière d'estimation du potentiel des sables lagonaires de Wallis, le processus d'étude de faisabilité est également en cours. Une reconnaissance a été réalisée sur 3 zones cibles (figure 3), par le biais d'une étude géophysique par sondage sismique continu du recouvrement sédimentaire superficiel du lagon de Wallis. Il a été utilisé de la sismique monotrace "petits fonds", haute définition. Quatre séquences sédimentaires superposées (ALLENBACH, 2000) ont été reconnues au sein du recouvrement superficiel du lagon de Wallis.

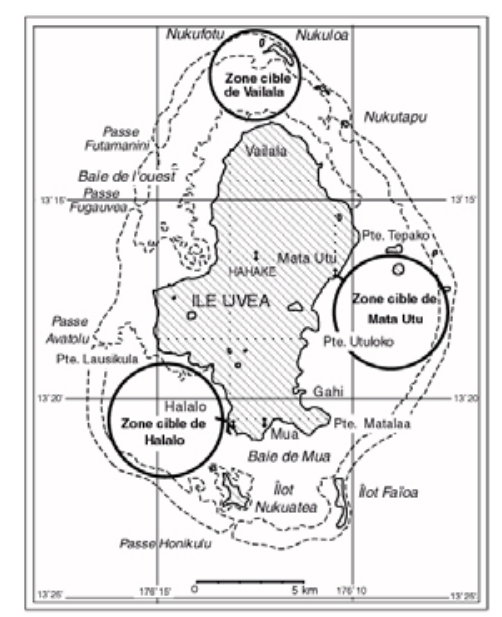

Figure 3. Les zones cibles reconnues par sondage sismique continu

Elles représentent, là où elles sont présentes, une épaisseur minimale de plusieurs dizaines de mètres de dépôts. Compte tenu d'une compaction et d'une cimentation croissantes probables en fonction de la profondeur, mais aussi des besoins du territoire en granulats, le potentiel volumétrique de la séquence superficielle semble être largement suffisant pour couvrir la demande exprimée, sans qu’il soit nécessaire de 
pousser plus loin l'analyse des séries plus profondes. Il convient pour aller plus avant dans l'analyse de cette variante, de choisir, en fonction des contraintes coutumières locales, les zones pouvant faire l'objet d'un consensus favorable à la décision d'une mise en exploitation. En aval des décisions coutumières devront être menées une étude de faisabilité socio-technico-économique du projet et une étude d'impact des zones identifiées comme favorables à l'extraction. Le matériel nécessaire à la mise en exploitation est disponible sur le territoire, mais le coût de production des granulats est aujourd'hui estimé voisin de celui des produits issus du concassage des matériaux basaltiques, ce qui pose les mêmes problèmes d'accès à la ressource par les populations locales. La démarche, si elle est menée à terme, peut toutefois générer la mise en place d'une activité salariée estimée à une dizaine de postes de travail, ce qui est loin d'être négligeable dans le contexte socio-économique de l'île où l'emploi salarié est essentiellement couvert par la fonction publique.

\subsection{L'importation de matériaux exogènes}

La communication se propose de se focaliser principalement sur l'utilisation potentielle des scories de nickel de l'usine SLN (Société le Nickel) implantée à Nouméa, en Nouvelle-Calédonie, comme produit de substitution aux matériaux naturels. Cette perspective peut répondre au cas particulier du territoire de Wallis et Futuna, mais également trouver d'autres champs d'application géographique dans une zone océannienne affectée par l'érosion littorale et déficitaire en granulats. La production de l'usine est de l'ordre de $1,5 \mathrm{M}$ de $\mathrm{m}^{3} / \mathrm{an}$. Les scories sont considérées comme inertes et sans danger pour l'environnement en Nouvelle-Calédonie car les péridotites qui correspondent au tiers des roches à l'affleurement induisent un bruit géochimique de fond très comparable à celui de la composition des crasses industrielles produites par Doniambo. Depuis 1940, la ville de Nouméa s'est ainsi agrandie de 320 ha gagnés sur la mer grâce aux remblais de scories et l'idée d'utiliser ces produits à Wallis et Futuna et en Océanie est logiquement apparue. Un groupe de travail réunissant l'industriel, l'ADECAL (Agence de Développement de la Nouvelle-Calédonie), des bureaux d'études et des scientifiques s'est constitué pour travailler dans ce sens, en vue d'une sensibilisation des acteurs économiques et politiques sur l'intérêt d'une telle démarche (KOLB et al, 2011). Deux verrous doivent être levés pour que cette valorisation devienne une réalité. Le premier est d'ordre économique et concerne le coût du transport vers les zones cibles. Des solutions techniques efficientes ont été identifiées pour assurer le chargement sur bateaux des scories à partir du site SLN de Doniambo à Nouméa et pour assurer le déchargement de ces produits sur des îles océaniennes dépourvues d'infrastructures portuaires. La faisabilité économique est assurée à partir d'un tonnage de $400000 \mathrm{t} / \mathrm{an}$. Le projet wallisien tourne autour d'un transport de l'ordre de $1 \mathrm{Mt}$ qui seraient utilisés pour poldériser une partie du littoral du district nord de l'île (zone de Hihifo) afin d'augmenter les surfaces disponibles en bord de mer à des fins 


\section{XII ${ }^{\text {èmes }}$ Journées Nationales Génie Côtier - Génie Civil \\ Cherbourg, 12-14 juin 2012}

d'usage collectif. Il intégrerait la mise en place de mini-structures portuaires (marinas, abri de pêche) et la construction des infrastructures collectives liées à l'organisation des mini-jeux du Pacifique de 2014, confiée au territoire. Le second est d'ordre environnemental et juridique. La convention de Bâle cadre juriquement ce type de procédure et il convient de démontrer que les scories ne sont pas dangereuses pour les écosystèmes où l'on souhaite les introduire. La composition chimique la plus classique des scories trouvée dans la littérature est proche de celle d'une péridotite appauvrie en Nickel $(\mathrm{Ni}=0,08 \%)$ et en Cobalt ( $\mathrm{Co}=0,01 \%)$ par le procédé pyro-métallurgique utilisé par l'usine. Ses principaux composants sont $\mathrm{SiO} 2$ (de 52 à $54 \%$ ), Mg0 (de 30 à $34 \%$ ) et $\mathrm{Fe} 0$ (de 9 à 11,5 \%). La distribution granulométrique des scories s'inscrit dans une plage comprise entre 0 et $15 \mathrm{~mm}$. Le d50 est de $2 \mathrm{~mm}$. La densité apparente est de 1,4 à 1,6 et la densité réelle comprise entre 2,8 et 3 . En matière d'impact environnemental, les résultats sur le potentiel de relargage de produits polluants des scories donnés par l'INERIS (2004) dans l'étude de référence la plus récente fournie lors de l'expertise sont sans ambiguité pour le groupe des scories de fusion (les produits dont l'exportation est envisagée) et sodo-calciques. "Ces scories présentent un faible potentiel polluant. Leurs teneurs en métaux et anions sur éluats et en composés organiques sur brut sont inférieures aux seuils définis pour l'acceptation en décharges pour déchets inertes. Ces caractéristiques permettent de les classer comme conformes aux critères pour la mise en décharge des déchets inertes..." Pour l'évaluation du comportement de la scorie de fusion au contact de l'eau de mer, le résultat est là encore sans ambiguité). Le test à l'eau salée "... a montré que la scorie de fusion lixiviée avec l'eau de mer présente un faible potentiel polluant et des teneurs conformes aux seuils fixés pour la mise en décharge de déchets inertes...".

Pour autant, le cas néo-calédonien n'est pas automatiquement transposable à d'autres territoires. Le principe de sécurité impose que soit réalisé l'ensemble des analyses permettant de vérifier que les résultats sont similaires pour d'autres environnements géologiques que celui de la Nouvelle-Calédonie. Cette précaution semble d'autant plus souhaitable que l'observation in situ montre que la scorie en remblais se consolide avec le temps, ce qui implique, soit une certaine mobilité des éléments qui la constitue, soit une transformation secondaire (carbonatation ?) au contact des éléments naturels (air, eau, eau de mer). Il paraît souhaitable de caractériser cette transformation secondaire et d'évaluer son implication dans l'évolution chimique du matériau dans des contextes différents. C'est le cas pour le territoire des Îles Wallis et Futuna dont la géologie est très différente (basaltes) de celle de la Nouvelle-Calédonie. Les scories constituent, en effet, une substance totalement exogène dont l'impact sur l'environnement doit être étudié précisément et cette précaution vaut pour l'ensemble des îles océaniennes visées par les projets d'exportation. L'expertise remise à la Préfecture en mars 2012 (ALLENBACH, 2012) demande que soit menée, avant toute décision d'importation à Wallis, une étude portant sur 4 points : (i) une caractérisation chimique fine de la scorie 
qui doit permettre d'avoir une meilleure connaissance du matériau solide : composition, phases minérales, spéciation des éléments potentiellement polluants; (ii) des essais de lixiviation sous différentes conditions de durée, de température, d'altération, de carbonatation; (iii) une modélisation géochimique visant à préciser les processus de dissolution/précipitation des différentes phases constitutives; (iiii) une comparaison avec les seuils règlementaires et les données de bruit de fond. Pour le territoire des Îles Wallis et Futuna, une telle étude, si elle est retenue, permettra aux décideurs politiques et économiques de se déterminer quant aux risques environnementaux encourrus par cette troisième variante, qui doit délivrer un produit dont le coût d'importation peut s'avérer compétitif par rapport à ceux des granulats fournis par les autres variantes. Pour les acteurs calédoniens qui s'intéressent tout particulièrement à d'éventuelles exportations vers les pays insulaires océaniens, le cas de Wallis et Futuna constitue un laboratoire grandeur nature qui peut permettre de lever les incertitudes techniques qui pèsent encore sur la démarche d'exportation envisagée.

\section{Références bibliographiques}

ALLENBACH M. (2003). Evolution naturelle et anthropisation du linéaire côtier de l'île de Wallis : une prise de conscience de l'impact environnemental des extractions de sable de plage. Collection "Îles et Archipels", n 32, pp 191-204

ALLENBACH M., HOIBIAN T. (2008). Enjeux, spécificités et perspectives de la gestion intégrée du littoral dans l'espace insulaire français du sud-ouest Pacifique. Européan Journal of Environmental and civil Engineering, Vol. 12, n 1-2, pp 15-34, doi:10.3166/ejece.12.15-34

ALLENBACH M. (2012). Bilan analytique des variantes granulats potentiellement utilisables sur le Territoire des Iles Wallis et Futuna. Note d'expertise Administration supérieure du Territoire/Service de l'Environnement, $63 \mathrm{p}$.

BANTOS S. (2011). Les sociétés ultramarines face au risque de montée du niveau marin. Quelles stratégies d'adaptation ? Thèse Paris IV, 546 p.

GIEC (2007). Rapport de synthèse "Changements climatiques 2007". http://www.ipcc.ch/pdf/assessment-report/ar4/syr/ar4_syr_fr.pdf

INERIS (2004). Caractérisation du potentiel polluant des échantillons de scories et de briques. Rapport pour la SLN, 22 pages + annexes.

KOLB P., CHU VAN D., ALLENBACH M. (2011). An example of an efficient management of industrial waste for coastal preservation in the PIC: use of nickel slag. Abstract PECC, Seminar 1: "Oceans at Risk: Protection from the ocean to the coast, sharing marine resources", Noumea, New Caledonia, November 22-24.

WORLICZEK E. (2011). Customary land tenure and the management of climate change and internal migration : The example of Wallis island. Land Tenure Journal. http://www.fao.org/nr/tenure/land-tenure-journal, pp 131-154 